\title{
A PROOF OF THE KAC-WAKIMOTO AFFINE DENOMINATOR FORMULA FOR THE STRANGE SERIES
}

\author{
DON ZAGIER
}

In [1], Kac and Wakimoto related various combinatorial formulas to the theory of root systems and representations of affine superalgebras. In particular, by specializing a conjectural affine denominator formula to the affine superalgebras associated to certain simple Lie superalgebras whose even parts are the simple Lie algebras of type $A_{m}$, they obtain a beautiful conjectural formula (Conjecture 7.2 of [1]) which contains as special cases many classical identities from the theory of modular forms and elliptic functions, as well as many new identities. The purpose of this paper is to prove this conjecture. A statement in elementary terms (with no reference to affine algebras or root systems) is given as Theorem 3 in $\S 3$.

The general case of the conjecture depends on $m+1$ parameters $q$ (which can be taken to be either a formal power series variable or a complex number of absolute value less than 1 ) and $x_{1}, \ldots, x_{m}$ (which are Laurent variables or nonzero complex numbers). Two specializations which are of particular interest, and which are singled out and stated as separate conjectures in [1], are the ones obtained by letting all variables $x_{j}$ tend to 1 or by taking $m=2$. These two cases will be stated and proved separately in $\S 1$ and $\S 2$, respectively, since they have simpler and more appealing statements than the general case and since the ideas used to prove them give the essence of the general proof. The first of these two results, Theorem 1 below, gives a formula for the number of representations of an arbitrary non-negative integer as a sum of $k$ triangular numbers when $k$ has the form $4 s^{2}$ (corresponding to $m=2 s-1$ ) or $4 s^{2}+4 s$ (corresponding to $m=2 s$ ) for some positive integer $s$, the cases $s=1$ being classical identities of Legendre for the number of representations of an integer as a sum of 4 or 8 triangular numbers. This theorem has also been proved by Milne [2]. The second, Theorem 2 below, gives an infinite product expansion for the sum $\sum_{j, k>0, k \text { odd }} q^{j k-1}(x y)^{1-j}\left(1-x^{j}\right)\left(1-y^{j}\right)\left(1-x^{j} y^{j}\right)([1]$, p. 451$)$.

The general identity, involving $q, x_{1}, \ldots, x_{m}$, belongs naturally to the theory of Jacobi forms, i.e., if we write $q=e^{2 \pi i \tau}$ and $x_{j}=e^{2 \pi i z_{j}}$ with $\tau \in \mathfrak{H}$ (upper half-plane) and $z_{j} \in \mathbb{C}$, then the functions appearing on its left- and righthand sides have modular transformation properties with respect to $\tau$ and elliptic transformation properties with respect to each $z_{j}$. The modular properties with

Received October 17, 2000. 
respect to $\tau$ will be used in the proof of the special case $z_{1}=\ldots=z_{m}=0$ in Theorem 1 (by contrast, Milne's proof avoids using modularity but instead makes heavy use of combinatorial arguments and identities from the theory of elliptic functions), and also in the proof of the general case, while the elliptic properties in the $z_{j}$ will be used in $\S 2$ and $\S 3$ in a disguised form (viz., in terms of the transformation properties under $x_{j} \rightarrow q^{2 n_{j}} x_{j}$ with $n_{j} \in \mathbb{Z}$, but without specifically introducing the logarithms $z_{j}$ ). Apart from basic facts from the theory of modular forms, the proofs are elementary.

Our notation is fairly standard. We will always use $\tau$ for a variable in $\mathfrak{H}$ and $q$ to denote $e^{2 \pi i \tau}$. We denote as usual by $\Gamma_{0}(2)$ and $\Gamma(2)$ the subgroups of $S L(2, \mathbb{Z})$ consisting of matrices $\left(\begin{array}{ll}a & b \\ c & d\end{array}\right)$ with $c$ even or with $b$ and $c$ even, respectively.

\section{Sums of triangular numbers}

For each positive integer $s$ we define two polynomials $P_{s}^{ \pm}\left(X_{1}, \ldots, X_{s}\right)$ in $s$ variables by

$$
\begin{aligned}
& P_{s}^{+}\left(X_{1}, \ldots, X_{s}\right)=\prod_{i=1}^{s} X_{i} \cdot \prod_{1 \leq i<j \leq s}\left(X_{i}^{2}-X_{j}^{2}\right)^{2}, \\
& P_{s}^{-}\left(X_{1}, \ldots, X_{s}\right)=\prod_{i=1}^{s} X_{i}^{3} \cdot \prod_{1 \leq i<j \leq s}\left(X_{i}^{2}-X_{j}^{2}\right)^{2} .
\end{aligned}
$$

Then for each positive integer $m$ we define

$$
\begin{aligned}
R_{s}^{+}(m) & =\sum_{\substack{r_{1} a_{1}+\cdots+r_{s} a_{s}=m \\
r_{i}, a_{i}>0, r_{i}, a_{i} \text { odd }}} P_{s}^{+}\left(a_{1}, \ldots, a_{s}\right), \\
R_{s}^{-}(m) & =\sum_{\substack{r_{1} a_{1}+\cdots+r_{s} a_{s}=m \\
r_{i}, a_{i}>0, r_{i} \text { odd }}} P_{s}^{-}\left(a_{1}, \ldots, a_{s}\right) .
\end{aligned}
$$

The following identity for sums of triangular numbers was stated as a conjecture in [1], p. 452.

Theorem 1. Denote by $\Delta_{k, n}$ the number of representations of a positive integer $n$ as a sum of $k$ triangular numbers. Then for all $s \geq 1, n \geq 0$ we have

$$
\Delta_{4 s^{2}, n}=\frac{R_{s}^{+}\left(2 n+s^{2}\right)}{c_{s}^{+}}, \quad \Delta_{4 s^{2}+4 s, n}=\frac{R_{s}^{-}\left(n+\frac{1}{2} s(s+1)\right)}{c_{s}^{-}},
$$

where the constants $c_{s}^{ \pm}$are given by

$$
c_{s}^{+}=4^{s(s-1)} s ! \prod_{j=1}^{2 s-1} j !, \quad c_{s}^{-}=2^{-s} s ! \prod_{j=1}^{2 s} j !
$$


Remark. The statement given by Kac and Wakimoto was slightly different in that in the definitions of $R_{s}^{ \pm}(m)$ it was assumed that $a_{1}>\cdots>a_{s}>0$ and the coefficients $c_{s}^{ \pm}$were smaller by a factor $s$ !, but the two statements are obviously equivalent since the $P_{s}^{ \pm}\left(a_{1}, \ldots, a_{s}\right)$ are symmetric in the $a_{i}$ and vanish if any two of them are equal.

Proof. For $\varepsilon= \pm 1$ let $M_{*}^{\varepsilon}(\Gamma(2))$ denote the subspace of modular forms on $\Gamma(2)$ satisfying $f(\tau+1)=\varepsilon f(\tau)$ (i.e., which have Fourier expansions involving only even or only odd powers of $q^{1 / 2}$ ). For each even integer $k \geq 2$ we have an Eisenstein series $g_{k}^{+} \in M_{k}^{-}(\Gamma(2))$ defined by

$$
g_{k}^{+}(\tau)=\sum_{\substack{r, a>0 \\ r, a \text { odd }}} a^{k-1} q^{r a / 2}=\sum_{\substack{n \geq 1 \\ n \text { odd }}} \sigma_{k-1}(n) q^{n / 2}=\frac{1}{2}\left(G_{k}\left(\frac{\tau}{2}\right)-G_{k}\left(\frac{\tau+1}{2}\right)\right),
$$

where $q=e^{2 \pi i \tau}$ and $G_{k}(\tau)=-\frac{1}{2} \zeta(1-k)+\sum_{n=1}^{\infty} \sigma_{k-1}(n) q^{n}$ denotes the standard Eisenstein series of weight $k$ and level 1. We define a map

$$
\Phi_{s}^{+}: X_{1} \cdots X_{s} \mathbb{C}\left[X_{1}^{2}, \ldots, X_{s}^{2}\right] \longrightarrow M_{*}^{(-1)^{s}}(\Gamma(2))
$$

by

$$
X_{1}^{k_{1}-1} \cdots X_{s}^{k_{s}-1} \longmapsto g_{k_{1}}^{+} \cdots g_{k_{s}}^{+} \quad\left(k_{i} \text { even, } k_{i} \geq 2\right) .
$$

Clearly $\Phi_{s}^{+}$is of degree $s$ (i.e., a monomial of degree $k-s$ goes to a modular form of weight $k$ ). In particular, since the polynomial $P_{s}^{+}$has degree $2 s^{2}-s$, the modular form $F_{s}^{+}:=\Phi_{s}^{+}\left(P_{s}^{+}\right)$has weight $2 s^{2}$. But $F_{s}^{+}(\tau)=\sum_{m>0} R_{s}^{+}(m) q^{m / 2}$ and clearly $R_{s}^{+}(m)=0$ for $m<s^{2}$ (the smallest integer representable as $\sum r_{i} a_{i}$ with $r_{i}$ and $a_{i}$ odd and all $a_{i}$ distinct is $1+3+\cdots+(2 s-1)=s^{2}$ ), so $F_{s}^{+}$ is an element of $M_{2 s^{2}}^{(-1)^{s}}(\Gamma(2))$ which vanishes to order at least $s^{2} / 2$ at infinity. This implies that $F_{s}^{+}$is a multiple of $\theta(\tau)^{4 s^{2}}$, where $\theta(\tau)=\sum_{n=0}^{\infty} q^{(2 n+1)^{2} / 8}=$ $q^{1 / 8}\left(1+q+q^{3}+q^{6}+\cdots\right)$ is the generating function for the triangular numbers. (To see this, note that $F_{s}^{+} / \theta^{4 s^{2}}$ is invariant under $\Gamma(2)$ and under $\tau \rightarrow \tau+1$ and hence under $\Gamma_{0}(2)$. But $\Gamma_{0}(2)$ has only two cusps. Since $\theta$ vanishes to order $1 / 8$ at infinity and is non-zero at the other cusp and in the upper half-plane, $F_{s}^{+} / \theta^{4 s^{2}}$ has no poles at finite points or cusps and hence must be constant.) This proves the first identity in (1) with some constant $c_{s}^{+}$. The value of this constant is seen by taking $n=0$ to be given by $c_{s}^{+}=R_{s}^{+}\left(s^{2}\right)=s ! P_{s}^{+}(1,3, \ldots, 2 s-1)$, which agrees with the first formula given in (2).

The second identity in (1) is similar. For each even integer $k \geq 4$ we have an Eisenstein series $g_{k}^{-} \in M_{k}^{+}(\Gamma(2))=M_{k}\left(\Gamma_{0}(2)\right)$ defined by

$$
g_{k}^{-}(\tau)=\sum_{\substack{r, a>0 \\ r \text { odd }}} a^{k-1} q^{r a}=G_{k}(\tau)-G_{k}(2 \tau)
$$


(Notice that $g_{2}^{-}(\tau)$, unlike $g_{2}^{+}(\tau)=\theta(\tau)^{4}$, is not modular.) We define a map of degree $s$

$$
\Phi_{s}^{-}: X_{1}^{3} \cdots X_{s}^{3} \mathbb{C}\left[X_{1}^{2}, \ldots, X_{s}^{2}\right] \longrightarrow M_{*}\left(\Gamma_{0}(2)\right)
$$

by

$$
X_{1}^{k_{1}-1} \cdots X_{s}^{k_{s}-1} \longmapsto g_{k_{1}}^{-} \cdots g_{k_{s}}^{-} \quad\left(k_{i} \text { even, } k_{i} \geq 4\right),
$$

and set $F_{s}^{-}=\Phi_{s}^{-}\left(P_{s}^{-}\right)$. Then $F_{s}^{-}(\tau)=\sum_{m>0} R_{s}^{-}(m) q^{m}$ has weight $2 s^{2}+2 s$ and vanishes to order $s(s+1) / 2$ (because the smallest integer representable as $\sum r_{i} a_{i}$ with $r_{i}$ odd and all $a_{i}$ distinct is $\left.1+2+\cdots+s=s(s+1) / 2\right)$, and hence is a multiple of $\theta(\tau)^{4 s^{2}+4 s}$ by the same argument as before, with the factor of proportionality now being given by $c_{s}^{-}=R_{s}^{-}(s(s+1) / 2)=s ! P_{s}^{-}(1,2, \ldots, s)$.

\section{The identity for $m=2$}

The identity below, the first unproved case of Conjecture 7.2 in [1], was stated there in detail on page 451 as a formal power series identity in three variables $q, x$ and $y$. We have changed the formulation slightly by introducing a third variable $z=(x y)^{-1}$ in order to render the symmetry coming from the Weyl group of $A_{2}$ more apparent.

Theorem 2. Let $q$ be a power series variable and define two series $\Theta_{i}(x)=$ $\Theta_{i}(q ; x) \in \mathbb{Z}\left[x, x^{-1}\right][[q]](i=0,1)$ by

$$
\begin{aligned}
& \Theta_{0}(x)=\sum_{n \in \mathbb{Z}}(-x)^{n} q^{n^{2}}=\prod_{n>0}\left(1-q^{2 n}\right)\left(1-q^{2 n-1} x\right)\left(1-q^{2 n-1} x^{-1}\right), \\
& \Theta_{1}(x)=\sum_{n \in \mathbb{Z}}(-x)^{n} q^{n^{2}-n}=\prod_{n>0}\left(1-q^{2 n}\right)\left(1-q^{2 n-2} x\right)\left(1-q^{2 n} x^{-1}\right) .
\end{aligned}
$$

Then for $x, y, z$ with $x y z=1$ we have

$$
-q \frac{\Theta_{1}^{\prime}(1) \Theta_{1}(x) \Theta_{1}(y) \Theta_{1}(z)}{\Theta_{0}(1) \Theta_{0}(x) \Theta_{0}(y) \Theta_{0}(z)}=\sum_{n=1}^{\infty}\left(1-x^{n}\right)\left(1-y^{n}\right)\left(1-z^{n}\right) \frac{q^{n}}{1-q^{2 n}} .
$$

Proof. We will work with complex rather than formal variables. Denote the left- and right-hand sides of (4) by $L(x, y, z)$ and $R(x, y, z)$, respectively. (The variable $q$ is considered as fixed and has been omitted from the notation, as was already done in equation (3).) The series $R(x, y, z)$ converges for $|q|<$ $|x|,|y|,|z|<|q|^{-1}$ and, by expanding the product $\left(1-x^{n}\right)\left(1-y^{n}\right)\left(1-z^{n}\right)$ as a sum of six monomials (two cancel), can be rewritten in the form

$$
R(x, y, z)=\Phi\left(x^{-1}\right)+\Phi\left(y^{-1}\right)+\Phi\left(z^{-1}\right)-\Phi(x)-\Phi(y)-\Phi(z),
$$

where

$$
\Phi(x)=\sum_{n=1}^{\infty} x^{n} \frac{q^{n}}{1-q^{2 n}} \quad\left(|x|<|q|^{-1}\right) .
$$


Clearly $\Phi(x)-\Phi\left(q^{2} x\right)=\sum_{n=1}^{\infty} x^{n} q^{n}=\frac{x q}{1-x q}$. This gives the meromorphic continuation of $\Phi(x)$ to all $x \in \mathbb{C}^{*}$, with simple poles at $x=q^{-n}(n=1,3,5, \ldots)$. It follows that the function $\Phi^{*}(x):=\Phi\left(x^{-1}\right)-\Phi(x)$ is also meromorphic in $\mathbb{C}^{*}$ with simple poles at $x=q^{n}\left(n \in \mathbb{Z}\right.$ odd) and satisfies $\Phi^{*}(x)-\Phi^{*}\left(q^{2} x\right)=1$. From this it follows in turn that $R(x, y, z)=\Phi^{*}(x)+\Phi^{*}(y)+\Phi^{*}(z)$ is invariant under $(x, y, z) \mapsto\left(q^{2 a} x, q^{2 b} y, q^{2 c} z\right)$ for any $a, b, c \in \mathbb{Z}$ with $a+b+c=0$. On the other hand, from the easy (and well-known) properties $\Theta_{0}\left(q^{2} x\right)=-q^{-1} x^{-1} \Theta_{0}(x)$, $\Theta_{1}\left(q^{2} x\right)=-x^{-1} \Theta_{1}(x)$ it follows that $\frac{\Theta_{1}}{\Theta_{0}}\left(q^{2} x\right)=q \frac{\Theta_{1}}{\Theta_{0}}(x)$ and hence that $L(x, y, z)$ has the same invariance property, so the quotient $R(x, y, z) / L(x, y, z)$ is a well-defined meromorphic function on the torus $\left(\mathbb{C} / q^{2 \mathbb{Z}}\right)_{\text {product }=1}^{3}$. Moreover, this function has no poles, since the poles of $R(x, y, z)$ at $x, y, z \in q^{\{\text {odd }\}}$ are killed by the zeros of $\Theta_{0}(x) \Theta_{0}(y) \Theta_{0}(z)$ and the zeros of $\Theta_{1}(x) \Theta_{1}(y) \Theta_{1}(z)$ are killed by the obvious vanishing of $R(x, y, z)$ at $(x=y=z=1$ and hence also) $x, y, z \in q^{\{\mathrm{even}\}}$. It follows that the quotient $R(x, y, z) / L(x, y, z)$ is a constant, and by letting $x, y, z \rightarrow 1$ we find the value of this constant to be equal to 1 .

\section{Remarks.}

1. We could have defined $\Theta_{0}$ and $\Theta_{1}$ using only the products in (3), since the expressions as sums were not used. For the same reason, we could have omitted the factor $\left(1-q^{2 n}\right)$ in both products, which cancels in (4) anyway (and in fact this was done in the statement of (4) given in [1]). We preferred to keep this factor since the definitions of the $\Theta$ 's as sums are simpler than the product formulas. Their equality, of course, is the Jacobi triple product formula.

2. From $\Phi(x)-\Phi\left(q^{2} x\right)=\frac{q x}{1-q x}\left(|x|<|q|^{-1}\right)$ we get $\Phi(x)=\sum_{n>0, n \text { odd }} \frac{q^{n} x}{1-q^{n} x}$ (all $x \in \mathbb{C}^{*} \backslash q^{\text {odd }\}}$ ) and hence $\Phi^{*}(x)=\Phi(1 / x)-\Phi(x)=x \Theta_{0}^{\prime}(x) / \Theta_{0}(x)$. The identity (4) can therefore be written

$$
-q \frac{\Theta_{1}^{\prime}(1) \Theta_{1}(x) \Theta_{1}(y) \Theta_{1}(z)}{\Theta_{0}(1) \Theta_{0}(x) \Theta_{0}(y) \Theta_{0}(z)}=x \frac{\Theta_{0}^{\prime}(x)}{\Theta_{0}(x)}+y \frac{\Theta_{0}^{\prime}(y)}{\Theta_{0}(y)}+z \frac{\Theta_{0}^{\prime}(z)}{\Theta_{0}(z)}
$$

and in this form can probably be identified (after multiplying through by the denominator of the left-hand side) as the derivative of a specialization of one of Riemann's theta-relations, though I have not checked this.

3. In the last step of the proof, we could have evaluated the constant by letting just $z$ tend to $q$ instead of letting all three variables $x, y, z$ tend to 1 , since it is easily checked that both $L\left(x, q x^{-1} z^{-1}, q^{-1} z\right)$ and $R\left(x, q x^{-1} z^{-1}, q^{-1} z\right)$ have a simple pole at $z=1$ with residue 1 (independent of $x$ ).

4. Dividing both sides of $(4)$ by $(1-x)(1-y)(1-z)$ and letting $x, y$ and $z$ tend to 1 gives

$$
\sum_{n=1}^{\infty} n^{3} \frac{q^{n}}{1-q^{2 n}}=q \frac{\Theta_{1}^{\prime}(1)^{4}}{\Theta_{0}(1)^{4}}=\theta(\tau)^{8}
$$


(because $\left.q^{1 / 4} \Theta_{1}^{\prime}(1) / \Theta_{0}(1)=q^{1 / 4} \prod_{n>1}\left(1-q^{n}\right)^{2(-1)^{n}}=\theta(\tau)^{2}\right)$, so we recover Legendre's formula that the number of representations of $m-1$ as a sum of 8 triangular numbers is the sum over all odd divisors $r$ of $m$ of $(m / r)^{3}$ (i.e., the case $s=1$ of the second formula in (1)).

\section{The general case}

The following theorem is an equivalent reformulation of Conjecture 7.2 of [1], but written in more elementary terms. The variables $x_{j}$ are $e^{-\alpha_{j}}$, where the $\alpha_{j}$ are the simple roots of $A_{m}$.

Theorem 3. Let $m$ be a positive integer and let $q$ and $x_{1}, \ldots, x_{m+1}$ be complex numbers satisfying $|q|<\left|x_{i} / x_{j}\right|<|q|^{-1}$ for all $i \neq j$. Set $s=\left[\frac{m+1}{2}\right]$. Then

$$
\begin{aligned}
& \sum_{k_{1} \geq \cdots \geq k_{s} \geq 0} \frac{q^{k_{1}+\cdots+k_{s}} x_{1}^{-\frac{m}{2}} x_{2}^{-\frac{m-2}{2}} \cdots x_{m+1}^{\frac{m}{2}}}{\prod_{i=1}^{s}\left(1-q^{2 k_{i}+m-2 i+2}\right)} \sum_{\sigma \in \mathfrak{S}_{m+1}} \varepsilon(\sigma) x_{\sigma(1)}^{k_{1}+\frac{m}{2}} x_{\sigma(2)}^{k_{2}+\frac{m-2}{2}} \cdots x_{\sigma(m+1)}^{-k_{1}-\frac{m}{2}} \\
& (5) \quad=\quad \Delta(q)^{2 s} \prod_{1 \leq i<j \leq m+1} \frac{\Theta_{1}\left(x_{j} / x_{i}\right)}{\Theta_{0}\left(x_{j} / x_{i}\right)}
\end{aligned}
$$

where $\Theta_{0}(x)$ and $\Theta_{1}(x)$ are defined as in $(3), \Delta(q)=q^{-1 / 8} \theta(\tau)=1+q+q^{3}+$ $q^{6}+\cdots$ is the generating function for triangular numbers, $\mathfrak{S}_{m+1}$ denotes the symmetric group on $m+1$ letters, $\varepsilon(\sigma)$ for $\sigma \in \mathfrak{S}_{m+1}$ denotes the sign of the permutation $\sigma$, and the exponents of the $x_{\sigma(i)}$ in the inner sum are $k_{i}+m / 2-i+1$ with the "extra" variables $k_{s+1}, \ldots, k_{m+1}$ defined by $k_{m+2-i}=-k_{i}$.

Proof. We will proceed as in the proof of Theorem 2 by showing that the ratio of the two sides of (5) is a constant independent of the $x_{i}$ and then evaluating the constant by considering the limit as all $x_{i}$ tend to 1 . We first introduce new variables $\lambda_{i}=2 k_{i}+m-2 i+2$. Then multiplying both sides of (5) by $q^{N / 2} x_{1}^{\frac{m}{2}} x_{2}^{\frac{m-2}{2}} \cdots x_{m+1}^{-\frac{m}{2}}$, where $N=\frac{1}{2}(m+(m-2)+\cdots+(m-2 s+2))=$ $\frac{2 s}{8}+\frac{1}{4}\left(\begin{array}{c}m+1 \\ 2\end{array}\right)$, we can rewrite this formula as

$$
\begin{aligned}
& \sum_{\substack{\lambda_{1}>\lambda_{2}>\ldots>\lambda_{s}>0 \\
\lambda_{1} \equiv \cdots \equiv \lambda_{s} \equiv m(\bmod 2)}} \frac{q^{\left(\lambda_{1}+\cdots+\lambda_{s}\right) / 2}}{\left(1-q^{\lambda_{1}}\right) \cdots\left(1-q^{\lambda_{s}}\right)} \operatorname{Alt}_{m+1}\left(\prod_{i=1}^{s}\left(\frac{x_{i}}{x_{m+2-i}}\right)^{\lambda_{i} / 2}\right) \\
& =\theta(\tau)^{2 s} \prod_{1 \leq i<j \leq m+1} F\left(x_{j} / x_{i}\right), \quad F(x):=q^{1 / 4} x^{-1 / 2} \frac{\Theta_{1}(x)}{\Theta_{0}(x)}
\end{aligned}
$$

where $\mathrm{Alt}_{m+1}$ denotes the alternating sum over all permutations of $x_{1}, \ldots, x_{m+1}$. We next observe (compare the remark following the statement of Theorem 1 in 
$\S 1)$ that the expression on the left-hand side of (5) is unchanged if we replace the condition " $\lambda_{1}>\lambda_{2}>\ldots>\lambda_{s}>0$ " by " $\lambda_{1}, \ldots, \lambda_{s}>0$ " and divide the sum by $s$ !, because the alternating sum is symmetric in the $\lambda_{i}$ (interchanging $\lambda_{i}$ and $\lambda_{j}$ corresponds to simultaneously interchanging $x_{i}$ with $x_{j}$ and $x_{m+2-i}$ with $x_{m+2-j}$, which is an even permutation) and vanishes if any two $\lambda_{i}$ 's are equal. In fact, we can replace this condition by " $\lambda_{1}, \ldots, \lambda_{s} \neq 0$ " and divide by $2^{s} s$ ! without changing anything, because the summand is also invariant under changing the sign of any $\lambda_{i}$ (this operation changes the sign of both $q^{\lambda_{i} / 2} /\left(1-q^{\lambda_{i}}\right)$ and of the alternating sum, since interchanging $x_{i}$ and $x_{m+2-i}$ is an odd permutation). The left-hand side of (6) can therefore be rewritten in the form

$$
\frac{1}{2^{s} s !} \sum_{\substack{\lambda_{1}, \ldots, \lambda_{s} \neq 0 \\ \lambda_{1} \equiv \cdots \equiv \lambda_{s} \equiv m(\bmod 2)}} \frac{q^{\left(\lambda_{1}+\cdots+\lambda_{s}\right) / 2}}{\left(1-q^{\lambda_{1}}\right) \cdots\left(1-q^{\lambda_{s}}\right)} \operatorname{Alt}_{m+1}\left(\prod_{i=1}^{s}\left(\frac{x_{i}}{x_{m+2-i}}\right)^{\lambda_{i} / 2}\right) .
$$

But in this new summation the various $\lambda_{i}$ run independently of one another over all even or odd non-zero integers, so the result to be proved can be rewritten as

$$
\begin{aligned}
\theta(\tau)^{2 s} & \prod_{1 \leq i<j \leq m+1} F\left(x_{i} / x_{j}\right) \\
= & \left\{\begin{array}{l}
\operatorname{Alt}_{2 s+1}^{*}\left(\Phi_{0}^{*}\left(x_{2 s+1} / x_{1}\right) \cdots \Phi_{0}^{*}\left(x_{s+2} / x_{s}\right)\right) \quad \text { if } m=2 s, \\
\operatorname{Alt}_{2 s}^{*}\left(\Phi_{1}^{*}\left(x_{2 s} / x_{1}\right) \cdots \Phi_{1}^{*}\left(x_{s+1} / x_{s}\right)\right) \quad \text { if } m=2 s-1,
\end{array}\right.
\end{aligned}
$$

where $\mathrm{Alt}_{m+1}^{*}$ denotes the alternating sum over the quotient of $\mathfrak{S}_{m+1}$ by the subgroup (isomorphic to $\mathfrak{S}_{s} \ltimes(\mathbb{Z} / 2 \mathbb{Z})^{s}$ ) of permutations preserving the set of unordered pairs $\left\{\left\{x_{i}, x_{m+2-i}\right\}\right\}_{1 \leq i \leq s}$, and

$$
\Phi_{i}^{*}(x)=\Phi_{i}\left(x^{-1}\right)-\Phi_{i}(x), \quad \Phi_{i}(x)=\sum_{\lambda>0, \lambda \equiv i \bmod 2} x^{\lambda / 2} \frac{q^{\lambda / 2}}{1-q^{\lambda}} \quad(i=0,1) .
$$

We consider the case when $m=2 s$ is even. (The case of odd $m$ is very similar and will be left to the reader.) The functions $\Phi_{0}(x)$ and $\Phi_{0}^{*}(x)$ are just the functions $\Phi(x)$ and $\Phi^{*}(x)$ which were already used in the proof of Theorem 2 . We saw there that $\Phi_{0}^{*}(x)$ has a meromorphic continuation to all $\mathbb{C}^{*}$, with simple poles at odd powers of $q$, and satisfies the functional equation $\Phi_{0}^{*}(x)-\Phi_{0}^{*}\left(q^{2} x\right)=1$. It follows that the right-hand side of $(7)$ is meromorphic in $\left(\mathbb{C}^{*}\right)^{m+1}$, is invariant under all translations $x_{i} \mapsto q^{2 n_{i}} x_{i}$ (adding 1 to one of the factors $\Phi_{0}^{*}\left(x_{i} / x_{j}\right)$ does not change the value of the alternating sum in (7) because interchanging the indices $i$ and $j$ is an odd permutation), has simple poles whenever two $x_{i}$ differ by an odd power of $q$, has no other poles, and vanishes whenever two $x_{i}$ differ by an even power of $q$ (by the antisymmetry in the $x_{i}$ and the periodicity with respect to $\left.x_{i} \mapsto q^{2 n_{i}} x_{i}\right)$. Since the function $F(x)$ is invariant under $x \mapsto q^{2} x$ and has simple poles at odd powers of $q$, simple zeros at even powers of $q$, and no 
other zeros or poles (because of the product expansions in (3)), these properties imply that the quotient of the right-hand side of (7) by the left-hand side is a holomorphic function on the compact complex manifold $\left(\mathbb{C}^{*} / q^{2 \mathbb{Z}}\right)^{m+1}$ and hence is a constant (for fixed $q$ ).

This proves equation (5) (for even $m$ ) up to a constant depending only on $q$. The evaluation of this constant can be performed in two ways, just as we already saw in the special case $m=2$ considered in $\S 2$. On the one hand, if we divide both sides of (5) by $\prod_{i<j}\left(x_{i}-x_{j}\right)$ and take the limit as all $x_{i}$ tend to 1 , then using the formula $F^{\prime}(1)=\theta(\tau)^{2}$ (cf. Remark 4 in $\S 2$ ) we find that the resulting identity is precisely Theorem 1 , which we have already proved. The calculation involved is not difficult and will be omitted here since it is precisely the one which was already given by Kac and Wakimoto to deduce the (then still conjectural) statement of Theorem 1 from that of Theorem 3. Alternatively, we can proceed as in Remark 3 of $\S 2$ by letting the quotient of two of the variables, say $x_{2 s+1}$ and $x_{1}$, tend to $q$ while the others remain generic. To be more specific, set $x_{2 s+1} / x_{1}=q e^{-t}$ and let $t \rightarrow 0$ in (7). Then, since the residue at $t=0$ of $\Phi_{0}^{*}\left(q e^{-t}\right)$ is equal to 1 , we find that the residue of the right-hand side is $\operatorname{Alt}_{2 s-1}^{*}\left(\Phi_{0}^{*}\left(x_{2 s} / x_{2}\right) \cdots \Phi_{0}^{*}\left(x_{s+2} / x_{s}\right)\right)$ (the only terms in $\operatorname{Alt}_{2 s+1}^{*}(\cdots)$ which have a pole are those where the variables $x_{1}$ and $x_{2 s+1}$ are paired with one another, and this reduces the $\mathrm{Alt}_{2 s+1}^{*}$ to an $\left.\mathrm{Alt}_{2 s-1}^{*}\right)$, and similarly that the residue on the left-hand side is $\theta(\tau)^{2 s-2} \prod_{2 \leq i<j \leq m} F\left(x_{i} / x_{j}\right)$ (the residue of $F\left(x_{2 s+1} / x_{1}\right)$ is $\theta(\tau)^{-2}$ and each product $F\left(x_{j} / x_{1}\right) F\left(x_{2 m+1} / x_{j}\right), 1<j<m+1$, equals 1$)$. The equality of the residues is therefore precisely the statement of Theorem 3 with $m$ replaced by $m-2$, so we can conclude by induction on $m$. This second argument is independent of Theorem 1 and therefore provides a second proof of that result, independent of the theory of modular forms.

\section{References}

[1] V. G. Kac and M. Wakimoto, Integrable highest weight modules over affine superalgebras and number theory, Lie Theory and Geometry in Honor of Bertram Kostant (Eds. J.L. Brylinski, R. Brylinski, V. Guillemin, and V. Kac), Progr. Math. 123 (1994), 415-456.

[2] S. C. Milne, Infinite families of exact sums of squares formulas, Jacobi elliptic functions, continued fractions, and Schur functions, Ramanujan J., to appear, (http://xxx.lanl.gov/abs/math.NT/0008068).

MaX-Planck-Institut für Mathematik, D-53111 Bonn, Germany.

E-mail address: don@mpim-bonn.mpg.de 